\title{
A highly efficient spectral-Galerkin method based on tensor product for fourth-order Steklov equation with boundary eigenvalue
}

\author{
Jing $A n^{1}$, Hai Bi ${ }^{1}$ and Zhendong LuO ${ }^{2 *}$ (D)
}

"Correspondence:
zhdluo@ncepu.edu.cn
${ }^{2}$ School of Mathematics and
Physics, North China Electric Power
University, No. 2, Bei Nong Road,
Changping District, Beijing, 102206,
China
Full list of author information is
available at the end of the article

\begin{abstract}
In this study, a highly efficient spectral-Galerkin method is posed for the fourth-order Steklov equation with boundary eigenvalue. By making use of the spectral theory of compact operators and the error formulas of projective operators, we first obtain the error estimates of approximative eigenvalues and eigenfunctions. Then we build a suitable set of basis functions included in $H_{0}^{1}(\Omega) \cap H^{2}(\Omega)$ and establish the matrix model for the discrete spectral-Galerkin scheme by adopting the tensor product. Finally, we use some numerical experiments to verify the correctness of the theoretical results.
\end{abstract}

MSC: $65 \mathrm{~N} 35 ; 65 \mathrm{~N} 30$

Keywords: fourth-order Steklov equation with boundary eigenvalue; spectral-Galerkin method; error estimates; tensor product

\section{Introduction}

Increasing attention has recently been paid to numerical approximations for Steklov equations with boundary eigenvalue, arising in fluid mechanics, electromagnetism, etc. (see, e.g., [1-11]). However, most existing work usually treated the second-order Steklov equations with boundary eigenvalue and there are relatively few articles treating fourth-order ones. The fourth-order Steklov equations with boundary eigenvalue also have been used in both mathematics and physics, for example, the main eigenvalues play a very key role in the positivity-preserving properties for the biharmonic-operator $\triangle^{2}$ under the border conditions $w=\Delta w-\chi w_{v}=0$ on $\partial \Theta$ (see [12]).

In this article, we take into account the following fourth-order Steklov equation with boundary eigenvalue:

$$
\begin{aligned}
& \triangle^{2} w=0, \quad \text { in } \Theta, \\
& w=0, \quad \text { on } \partial \Theta, \\
& \triangle w=\chi w_{v}, \quad \text { on } \partial \Theta,
\end{aligned}
$$

(c) 2016 An et al. This article is distributed under the terms of the Creative Commons Attribution 4.0 International License (http://creativecommons.org/licenses/by/4.0/), which permits unrestricted use, distribution, and reproduction in any medium, provided you give appropriate credit to the original author(s) and the source, provide a link to the Creative Commons license, and indicate if changes were made. 
where $\Theta \subset \mathbb{R}^{d}(d=2,3)$ is a rectangular domain or a cuboid domain, $w_{v}$ represents the outer normal derivative of $w$ on $\partial \Theta$.

A conforming finite element method was first studied and a bound for the exact eigenvalues was provided in [13] for (1.1)-(1.3). Compared with finite element methods, spectral methods have the characteristics of high accuracy (see [14]). Especially, as far as we know, there is no work at all on spectral methods for the fourth-order Steklov equations with boundary eigenvalue, which is different from the equation and technique in [15]. So, this article aims to build a successful spectral-Galerkin formulation for the fourthorder Steklov equation with boundary eigenvalue. The article includes at least the following three features.

(1) We adopt the generalized Jacobian polynomial to deduce in detail the error formula of the high dimensional projective operator associated with the fourth-order Steklov equation with boundary eigenvalue. Then by employing the spectral method of compact operators, we obtain the satisfactory error formulas of approximative eigenvalues and eigenfunctions.

(2) We formulate a suitable set of basis functions and build the matrix formulation for the discrete variational scheme by means of the tensor product. Especially, we combine a set of basis function in spectral space included in $H_{0}^{2}(\Theta)$ with two basis functions in spectral space included in $H_{0}^{1}(\Theta)$ to formulate the basis functions. In this way, the matrix formulation obtained is sparse so that it can easily and efficiently be solved.

(3) We not only present the numerical example in the two-dimensional domain for the equation, but we also provide the three-dimensional one which has not been reported as far as we know. The numerical results explain the effectiveness of our approach.

The remainder of this article is arranged as follows. Section 2 provides some preparations. Section 3 provides the error formulas of the projective-operator and the spectralGalerkin approximate solutions. In Section 4, we establish the matrix model based on the tensor product for the discrete variational model such that it can easily be solved. In Section 5, we enumerate some numerical examples to confirm the accuracy and efficiency of the theoretical results. Finally, in Section 6, we give main conclusions.

\section{Some preparations}

Let $H^{s}(\Theta)$ and $H^{s}(\partial \Theta)$ be the standard Sobolev space on $\Theta$ and $\partial \Theta$ with integer order $s$, respectively. $H^{0}(\Theta)=L^{2}(\Theta), H^{0}(\partial \Theta)=L^{2}(\partial \Theta), H_{0}^{1}(\Theta)=\left\{v \in H^{1}(\Theta): v=0\right.$ on $\left.\partial \Theta\right\}$. The norm in $H^{s}(\Theta)$ and $H^{s}(\partial \Theta)$ are represented by $\|\cdot\|_{s}$ and $\|\cdot\|_{s, \partial \Theta}$, respectively. Throughout this article, $C$ is a generic positive constant independent of the degree $N$ of polynomials that may be unequal at the various places.

Put $V=H^{2}(\Theta) \cap H_{0}^{1}(\Theta)$. The variational formulation for (1.1)-(1.3) is stated as follows. Seek $\chi \in \mathbb{R}$ and nonzero $w \in V$ that satisfy

$$
a(w, v)=\chi b(w, v), \quad \forall v \in V,
$$

where $a(w, v)=\int_{\Theta} \Delta w \Delta v d x, b(w, v)=\int_{\partial \Theta} w_{v} v_{v} d s$.

The source equation corresponding to (2.1) is denoted in the following form.

Seek $w \in V$ that satisfies

$$
a(w, v)=b(f, v), \quad \forall v \in V .
$$


It is obvious that $a(\cdot, \cdot)$ is a continuous, symmetric, and $V$-elliptic bilinear function on $V \times V$ and $b(f, \cdot)$ is a linear and continuous function on $V$ (see [13]). Thus, we can define the norm in $V$ by $\|\cdot\|_{a}=\sqrt{a(\cdot, \cdot)}$ equivalent with the norm $\|\cdot\|_{2}$ in $H^{2}(\Theta)$. Further, by the Lax-Milgram theorem, for equation (2.2) there exists a unique solution. Therefore, for $g \in V$, the source equation (2.2) may determine an operator $\Gamma: V \rightarrow V$ that satisfies

$$
a(\Gamma g, v)=b(g, v), \quad \forall v \in V
$$

From [16] and (2.1), we obtain the following equivalent operator formula:

$$
\Gamma w=\frac{1}{\chi} w
$$

It follows from Lemma 2.2 in [13] that the operator $\Gamma: V \rightarrow V$ is self-adjoint and compact. Then, from the spectral method of compact operators (see, e.g., [16, 17]), we deduce that all eigenvalues of $\Gamma$ are real and finite multiple numbers, which are increasingly arranged as follows:

$$
0<\chi_{1} \leq \chi_{2} \leq \chi_{3} \leq \cdots \nearrow+\infty
$$

The eigenfunctions with respect to two disparate eigenvalues of $\Gamma$ must be orthometric. Therefore, there must be a standard orthometric basis in the eigenspace for the same eigenvalue. Thus, we can formulate a complete orthometric system of $V$ by means of the eigenfunctions of $\Gamma$ with respect to $\left\{\chi_{j}\right\}$ as follows:

$$
w_{1}, w_{2}, \ldots, w_{j}, \ldots
$$

Next, we specify some notations which will appear in the sequel. For simplicity, we consider $\Theta=I^{d}(d=2,3)$ where $I=(-1,1)$. Let $L_{n}(x)$ represent the Legendre polynomial of degree $n$ and let

$$
P_{N}=\operatorname{span}\left\{L_{0}(x), L_{1}(x), \ldots, L_{N}(x)\right\}, \quad S_{N}=P_{N} \cap H_{0}^{1}(I), \quad X_{N}=S_{N}^{d} .
$$

Then the spectral-Galerkin discrete formulation of (2.1) is denoted in the following form. Seek $\chi_{N} \in \mathbb{R}$ and nonzero $w_{N} \in X_{N}$ that satisfy

$$
a\left(w_{N}, v_{N}\right)=\chi_{N} b\left(w_{N}, v_{N}\right), \quad \forall v_{N} \in X_{N} .
$$

The discrete formulation with respect to (2.2) can be denoted in the following form.

Seek $w_{N} \in X_{N}$ that satisfies

$$
a\left(w_{N}, v\right)=b(f, v), \quad \forall v \in X_{N} .
$$

Similarly, it follows from the Lax-Milgram theorem that for (2.6) there exists one and only one solution. Further, for $g \in V$ there is the operator $\Gamma_{N}: V \rightarrow X_{N}$ that satisfies

$$
a\left(\Gamma_{N} g, v\right)=b(g, v), \quad \forall v \in X_{N} .
$$


From [16] and (2.5), we obtain the following equivalent operator formula in operator form:

$$
\Gamma_{N} w_{N}=\frac{1}{\chi_{N}} w_{N}
$$

It is obvious that the rank of the operator $\Gamma_{N}: V \rightarrow X_{N}$ is finite.

Define a projection-operator $\Pi_{N}^{1,0}: V \rightarrow X_{N}$ by

$$
a\left(w-\Pi_{N}^{1,0} w, v\right)=0, \quad \forall w \in V, v \in X_{N} .
$$

Lemma 2.1 If $\Gamma$ and $\Gamma_{N}$ be bounded linear operators determined by (2.3) and (2.7), respectively, then we have the following equality:

$$
\Gamma_{N}=\Pi_{N}^{1,0} \Gamma
$$

Proof For $\forall w \in V, v \in X_{N}$, we have

$$
a\left(\Pi_{N}^{1,0} \Gamma w-\Gamma_{N} w, v\right)=a\left(\Pi_{N}^{1,0} \Gamma w-\Gamma w, v\right)+a\left(\Gamma w-\Gamma_{N} w, v\right)=0
$$

By taking $v=\Pi_{N}^{1,0} \Gamma w-\Gamma_{N} w$ in (2.10), we obtain

$$
a\left(\Pi_{N}^{1,0} \Gamma w-\Gamma_{N} w, \Pi_{N}^{1,0} \Gamma w-\Gamma_{N} w\right)=0 .
$$

Because $a(\cdot, \cdot)$ is $V$-elliptic, we immediately obtain $\Gamma_{N}=\Pi_{N}^{1,0} \Gamma$.

It is obvious that the rank of the operator $\left.\Gamma_{N}\right|_{X_{N}}: X_{N} \rightarrow X_{N}$ is finite. Therefore, the eigenvalues of (2.5) may be increasingly arranged as follows:

$$
0<\chi_{1 N} \leq \chi_{2 N} \leq \chi_{3 N} \leq \cdots \leq \chi_{K N} \quad\left(K=\operatorname{dim} X_{N}\right)
$$

\section{Error analysis}

We will devote this section to estimating the errors between $\chi_{N}$ and $\chi$, and $w_{N}$ and $w$.

Lemma 3.1 If $(\chi, w)$ and $\left(\chi_{N}, w_{N}\right)$ are the kth eigenpairs of (2.1) and (2.5), respectively, then we have

$$
\chi_{N}-\chi=\frac{\left\|w_{N}-w\right\|_{a}^{2}}{\left\|\left(w_{N}\right)_{v}\right\|_{L^{2}(\partial \Theta)}^{2}}-\chi \frac{\left\|\left(w_{N}-w\right)_{v}\right\|_{L^{2}(\partial \Theta)}^{2}}{\left\|\left(w_{N}\right)_{v}\right\|_{L^{2}(\partial \Theta)}^{2}} .
$$

Proof From (2.1), we have

$$
\begin{aligned}
& a\left(w_{N}-w, w_{N}-w\right)-\chi b\left(w_{N}-w, w_{N}-w\right) \\
&=a\left(w_{N}, w_{N}\right)-2 a\left(w_{N}, w\right)+a(w, w)-\chi b\left(w_{N}, w_{N}\right)+2 \chi b\left(w_{N}, w\right) \\
&-\chi b(w, w)=a\left(w_{N}, w_{N}\right)-2 \chi b\left(w_{N}, w\right)+\chi b(w, w) \\
&-\chi b\left(w_{N}, w_{N}\right)+2 \chi b\left(w_{N}, w\right)-\chi b(w, w) \\
&= a\left(w_{N}, w_{N}\right)-\chi b\left(w_{N}, w_{N}\right) .
\end{aligned}
$$


Dividing $b\left(w_{N}, w_{N}\right)$ in both sides of the above equation and using (2.5), we obtain

$$
\chi_{N}-\chi=\frac{\left\|w_{N}-w\right\|_{a}^{2}}{\left\|\left(w_{N}\right)_{v}\right\|_{L^{2}(\partial \Theta)}^{2}}-\chi \frac{\left\|\left(w_{N}-w\right)_{\nu}\right\|_{L^{2}(\partial \Theta)}^{2}}{\left\|\left(w_{N}\right)_{\nu}\right\|_{L^{2}(\partial \Theta)}^{2}}
$$

Put

$$
\eta_{N}=\sup _{w \in V,\|w\|_{a}=1} \inf _{v \in X_{N}}\|\Gamma w-v\|_{a}
$$

It is obvious that

$$
\eta_{N} \rightarrow 0 \quad(N \rightarrow \infty)
$$

Theorem 3.1 We have

$$
\left\|\Gamma-\Gamma_{N}\right\|_{a} \rightarrow 0 \quad(N \rightarrow \infty)
$$

Proof With the norm of the operator, we obtain

$$
\begin{aligned}
\left\|\Gamma-\Gamma_{N}\right\|_{a} & =\sup _{w \in V,\|w\|_{a}=1}\left\|\left(\Gamma-\Gamma_{N}\right) w\right\|_{a} \\
& =\sup _{w \in V,\|w\|_{a}=1}\left\|\Gamma w-\Pi_{N}^{1,0} \Gamma w\right\|_{a} \\
& =\sup _{w \in V,\|w\|_{a}=1} \inf _{v \in X_{N}}\|\Gamma w-v\|_{a} \\
& =\eta_{N} \rightarrow 0 \quad(N \rightarrow \infty) .
\end{aligned}
$$

Let $M(\chi)$ represent the eigenfunction subspace with respect to the eigenvalue $\chi$ of (2.1).

Theorem 3.2 If $(\chi, w)$ and $\left(\chi_{N}, w_{N}\right)$ are the kth eigenpairs of (2.1) and (2.5), respectively, then we have

$$
\begin{aligned}
& \left\|w-w_{N}\right\|_{a} \leq \sup _{w \in M(\chi),\|w\|_{a}=1} \frac{C}{\chi}\left\|w-\Pi_{N}^{1,0} w\right\|_{a} \\
& \chi_{N}-\chi \leq \sup _{w \in M(\chi),\|w\|_{a}=1} \frac{C}{\chi^{2}} \frac{\left\|w-\Pi_{N}^{1,0} w\right\|_{a}^{2}}{\left\|\left(w_{N}\right)_{v}\right\|_{L^{2}(\partial \Theta)}^{2}}
\end{aligned}
$$

Proof From Theorem 3.1, we deduce that $\left\|\Gamma-\Gamma_{N}\right\|_{a} \rightarrow 0(N \rightarrow \infty)$. Thus, from Theorem 7.4 in [16], we obtain

$$
\left\|w-w_{N}\right\|_{a} \leq C\left\|\left.\left(\Gamma-\Gamma_{N}\right)\right|_{M(\chi)}\right\|_{a}
$$

Therefore, for any $w \in M(\chi)$ that satisfies $\|w\|_{a}=1$, we deduce that

$$
\begin{aligned}
& \left\|\left(\Gamma-\Gamma_{N}\right) w\right\|_{a}=\left\|\Gamma w-\Pi_{N}^{1,0} \Gamma w\right\|_{a}=\frac{1}{\chi}\left\|w-\Pi_{N}^{1,0} w\right\|_{a}, \\
& \left\|\left.\left(\Gamma-\Gamma_{N}\right)\right|_{M(\chi)}\right\|_{a}=\sup _{w \in M(\chi),\|w\|_{a}=1}\left\|\left(\Gamma-\Gamma_{N}\right) w\right\|_{a} .
\end{aligned}
$$


Combining (3.7) with the above two inequalities yields the desired conclusion (3.5). By using Lemma 3.1, we obtain

$$
\chi_{N}-\chi \leq \frac{\left\|w_{N}-w\right\|_{a}^{2}}{\left\|\left(w_{N}\right)_{v}\right\|_{L^{2}(\partial \Theta)}^{2}} .
$$

Combining the above inequality with (3.5) yields (3.6).

Denote by $J_{n}^{\sigma, \kappa}(x)$ the Jacobi polynomials that are orthometric about the Jacobi weight function $\omega^{\sigma, \kappa}(x):=(1-x)^{\sigma}(1+x)^{\kappa}$ over $I:=(-1,1)$, i.e.,

$$
\int_{-1}^{1} J_{n}^{\sigma, \kappa}(x) J_{m}^{\sigma, \kappa}(x) \omega^{\sigma, \kappa}(x) d x=\gamma_{n}^{\sigma, \kappa} \delta_{m n}
$$

where $\gamma_{n}^{\sigma, \kappa}=\left\|J_{n}^{\sigma, \kappa}\right\|_{\omega^{\sigma, \kappa}}^{2}$.

Since for $\sigma \leq-1$ and/or $\kappa \leq-1$, the function $\omega^{\sigma, \kappa}(x)$ is not in $L^{1}(I)$, it cannot be used as a general weight function. However, the usual Jacobi polynomials only include the case where $\sigma>-1$ and $\kappa>-1$. Thus, it is necessary to extend the definition of the usual Jacobi polynomials to the following cases where one or both negative integer(s)

$$
J_{n}^{k, l}(x)= \begin{cases}(1-x)^{-k}(1+x)^{-l} J_{n-n_{0}}^{-k,-l}(x), & \text { if } k, l \leq-1, \\ (1-x)^{-k} J_{n-n_{0}}^{-k, l}(x), & \text { if } k \leq-1, l>-1, \\ (1+x)^{-l} J_{n-n_{0}}^{k,-l}(x), & \text { if } k>-1, l \leq-1,\end{cases}
$$

where $n_{0} \leq n$ and

$$
n_{0}= \begin{cases}-(k+l), & \text { if } k, l \leq-1 \\ -k, & \text { if } k \leq-1, l>-1 \\ -l, & \text { if } k>-1, l \leq-1\end{cases}
$$

Let $\hat{J}_{n}^{k, l}(x)$ be the normalized Jacobi polynomials that satisfy

$$
\int_{-1}^{1} \hat{J}_{n}^{k, l}(x) \hat{J}_{m}^{k, l}(x) \omega^{k, l}(x) d x=\delta_{m n}
$$

Now, the $d$-dimensional $(d=2,3)$ generalized Jacobi polynomial tensors and weight functions are defined by

$$
\boldsymbol{J}_{\boldsymbol{n}}^{\boldsymbol{k}, \boldsymbol{l}}(\mathbf{x})=\prod_{j=1}^{d} \hat{j}_{n_{j}}^{k_{j}, l_{j}}\left(x_{j}\right), \quad \boldsymbol{\omega}^{k, l}(x)=\prod_{j=1}^{d} \omega^{k_{j}, l_{j}}\left(x_{j}\right)
$$

where $\boldsymbol{n}=\left(n_{1}, n_{2}, \ldots, n_{d}\right) \in \mathbb{N}^{d}, \boldsymbol{k}=\left(k_{1}, k_{2}, \ldots, k_{d}\right), \boldsymbol{I}=\left(l_{1}, l_{2}, \ldots, l_{d}\right)$ with $n_{j} \geq 2, k_{j}=l_{j}=-1$ $(j=1,2, \ldots, d)$. Then the $d$-dimensional generalized Jacobi polynomial tensors $\boldsymbol{J}_{\boldsymbol{n}}^{\boldsymbol{k} \boldsymbol{I}}(\boldsymbol{x})$ constitute a complete orthometric basis in $L_{\mathbf{w}^{k, l}}^{2}\left(I^{d}\right)$. Thus, the $d$-dimensional polynomial subspace of degree $N$ can be defined by

$$
\mathbf{Q}_{N}^{\boldsymbol{k}, \boldsymbol{l}}:=\operatorname{span}\left\{\boldsymbol{J}_{\boldsymbol{n}}^{\boldsymbol{k}, \boldsymbol{l}}(\boldsymbol{x}):|\boldsymbol{n}|_{\infty} \leq N\right\},
$$


where $|\boldsymbol{n}|_{\infty}=\max _{1 \leq j \leq d} n_{j}$. Further, the orthometric projection $\tilde{\Pi}_{N}^{\boldsymbol{k}, \boldsymbol{l}}: L_{\mathbf{w}^{\boldsymbol{k}, \boldsymbol{l}}}^{2}\left(I^{d}\right) \rightarrow Q_{N}^{\boldsymbol{k}, \boldsymbol{l}}$ is defined by

$$
\int_{I^{d}}\left(\tilde{\Pi}_{N}^{\boldsymbol{k}, \boldsymbol{l}} w-w\right) v_{N} \boldsymbol{\omega}^{\boldsymbol{k}, \boldsymbol{l}} d \mathbf{x}=0, \quad \forall v_{N} \in Q_{N}^{\boldsymbol{k}, \boldsymbol{l}}
$$

Finally, the $d$-dimensional Jacobi-weighted Sobolev space, which is an extension of the one-dimensional case, is defined by

$$
B_{\boldsymbol{k}, I}^{m}\left(I^{d}\right):=\left\{\boldsymbol{w}: \partial_{\boldsymbol{x}}^{\boldsymbol{m}} \boldsymbol{w} \in L_{\boldsymbol{w}^{\boldsymbol{k}+\boldsymbol{m}, l+\boldsymbol{m}}}^{2}\left(I^{d}\right), 0 \leq|\boldsymbol{m}|_{1} \leq m\right\}, \quad \forall m \in \mathbb{N},
$$

whose semi-norm and norm are, respectively, as follows:

$$
\begin{aligned}
& |\boldsymbol{w}|_{B_{\boldsymbol{k}, I^{m}}^{m}\left(I^{d}\right)}:=\left(\sum_{j=1}^{d}\left\|\partial_{x_{j}}^{m} \boldsymbol{w}\right\|_{\boldsymbol{w}^{\boldsymbol{k}+m \boldsymbol{e}_{j}, \boldsymbol{I}+m \boldsymbol{e}_{,}, I^{d}}}^{2}\right)^{\frac{1}{2}}, \\
& \|\boldsymbol{w}\|_{B_{\boldsymbol{k}, I^{m}\left(I^{d}\right)}}:=\left(\sum_{0 \leq|\boldsymbol{m}|_{1} \leq m}\left\|\partial_{\boldsymbol{x}}^{\boldsymbol{m}} \boldsymbol{w}\right\|_{\boldsymbol{w}^{\boldsymbol{k}+\boldsymbol{m}, I^{+}+\boldsymbol{m}_{,} I^{d}}}^{2}\right)^{\frac{1}{2}},
\end{aligned}
$$

where $\boldsymbol{m}=\left(m_{1}, m_{2}, \ldots, m_{d}\right) \in \mathbb{N}^{d},|\boldsymbol{m}|_{1}=\sum_{j=1}^{d} m_{j}, \partial_{\boldsymbol{x}}^{\boldsymbol{m}} \boldsymbol{w}=\partial_{x_{1}}^{m_{1}} \ldots \partial_{x_{d}}^{m_{d}} \boldsymbol{w}$, and $\boldsymbol{e}_{j}$ is the $j$ th unit vector of $\mathbb{R}^{d}$.

The following lemma is directly obtained from Theorem 8.1 and Remark 8.14 in [14].

Lemma 3.2 For any $\boldsymbol{w} \in B_{\boldsymbol{k}, I}^{m}\left(I^{d}\right)$ and $2 \leq m \leq N+1$, we have the following error formula:

$$
\left|\tilde{\Pi}_{N}^{\boldsymbol{k}, \boldsymbol{l}} \boldsymbol{w}-\boldsymbol{w}\right|_{B_{\boldsymbol{k}, I^{2}\left(I^{d}\right)}} \leq c \sqrt{\frac{(N-m) !}{(N-2) !}}(N+m)^{\frac{2-m}{2}}|\boldsymbol{w}|_{B_{\boldsymbol{k}, I^{m}}\left(I^{d}\right)}
$$

where $c \simeq \sqrt{2}$ for $N \gg 1$.

Before we proceed with the error formulas, it is important to make the following obser-

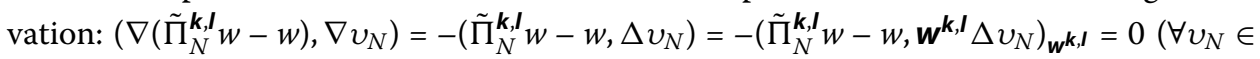
$\left.X_{N}\right)$. Thus, $\tilde{\Pi}_{N}^{\boldsymbol{k}, \boldsymbol{l}}$ is also an orthometric projection from $V$ into $X_{N}$.

Theorem 3.3 If $(\chi, w)$ and $\left(\chi_{N}, w_{N}\right)$ are the kth eigenpairs of (2.1) and (2.5), respectively, then, for any $w \in B_{\boldsymbol{k}, l}^{m}\left(I^{d}\right)$, we have

$$
\begin{aligned}
& \left\|w-w_{N}\right\|_{a} \leq \sup _{w \in M(\chi),\|w\|_{a}=1} \frac{C}{\chi} N^{2-m}|w|_{B_{\boldsymbol{k}, I^{m}}^{m}\left(I^{d}\right)}, \\
& \chi_{N}-\chi \leq \sup _{w \in M(\chi),\|w\|_{a}=1} \frac{C}{\chi^{2}\left\|\left(w_{N}\right)_{v}\right\|_{L^{2}(\partial \Theta)}^{2}} N^{2(2-m)}|w|_{B_{\boldsymbol{k}, I^{m}}^{m}\left(I^{d}\right)}^{2}, \quad 2 \leq m \leq N+1 .
\end{aligned}
$$

Proof From (2.9) and the properties of $a(w, v)$, we can derive

$$
\begin{aligned}
\left\|w-\Pi_{N}^{1,0} w\right\|_{a}^{2} & =a\left(w-\Pi_{N}^{1,0} w, w-\Pi_{N}^{1,0} w\right) \\
& =\inf _{\phi_{N} \in X_{N}} a\left(w-\phi_{N}, w-\phi_{N}\right)
\end{aligned}
$$




$$
\begin{aligned}
& \leq C \inf _{\phi_{N} \in X_{N}}\left|w-\phi_{N}\right|_{H^{2}\left(I^{d}\right)}^{2} \\
& \leq C\left|w-\tilde{\Pi}_{N}^{\boldsymbol{k}, \boldsymbol{l}} w\right|_{H^{2}\left(I^{d}\right)}^{2} \\
& \leq C\left|\tilde{\Pi}_{N}^{\boldsymbol{k}, \boldsymbol{l}} w-w\right|_{B_{\boldsymbol{k}, I^{2}\left(I^{d}\right)}^{2} .}
\end{aligned}
$$

Thus, from Lemma 3.2, we obtain

$$
\left\|w-\Pi_{N}^{1,0} w\right\|_{a}^{2} \leq C\left|\tilde{\Pi}_{N}^{\boldsymbol{k}, \boldsymbol{l}} w-w\right|_{B_{\boldsymbol{k}, I^{2}\left(I^{d}\right)}^{2}} \leq C \frac{(N-m) !}{(N-2) !}(N+m)^{2-m}|w|_{B_{\boldsymbol{k}, I^{m}}^{m}\left(I^{d}\right)}^{2} .
$$

By combining Theorem 3.2 and (3.226) in [14], we immediately obtain the desired conclusion.

\section{Efficient implementation of the spectral-Galerkin solutions}

We will devote this section to providing the approach to solve equation (2.5) efficiently. To this end, we first formulate a basis of normal orthometric functions for $X_{N}$.

Let $\phi_{k}(x)=\frac{1}{\sqrt{2(2 k+3)^{2}(2 k+5)}}\left(L_{k}(x)-\frac{2(2 k+5)}{2 k+7} L_{k+2}(x)+\frac{2 k+3}{2 k+7} L_{k+4}\right)(k=0,1, \ldots, N-4), \phi_{N-3}(x)=$ $L_{0}(x)-L_{2}(x)$, and $\phi_{N-2}(x)=L_{1}(x)-L_{3}(x)$. It is obvious that $\left\{\phi_{k}\right\}_{k=0}^{N-2}$ constitutes a basis for $X_{N}$.

From Lemma 3.1 in [18] and the orthogonality of Legendre polynomial, we immediately obtain the following lemma.

Lemma 4.1 If $a_{k j}=\left(\phi_{j}^{\prime \prime}, \phi_{k}^{\prime \prime}\right), b_{k j}=\left(\phi_{j}, \phi_{k}\right)$, and $c_{k j}=\left(\phi_{j}^{\prime}, \phi_{k}^{\prime}\right)$, then we have the following results.

(1) When $k, j=0,1, \ldots, N-4, a_{k j}=\delta_{k j}$ and the only nonzero elements of $b_{k j}$ and $c_{k j}$ are

$$
\begin{aligned}
& b_{k k}=d_{k}^{2}\left(e_{k}+h_{k}^{2} e_{k+2}+g_{k}^{2} e_{k+4}\right), \\
& b_{k, k+2}=b_{k+2, k}=d_{k} d_{k+2}\left(h_{k} e_{k+2}+g_{k} h_{k+2} e_{k+4}\right), \\
& b_{k, k+4}=b_{k+4, k}=d_{k} d_{k+4} g_{k} e_{k+4}, \\
& c_{k k}=-2(2 k+3) d_{k}^{2} h_{k}, \\
& c_{k, k+2}=c_{k+2, k}=-2(2 k+3) d_{k} d_{k+2},
\end{aligned}
$$

where $e_{k}=\frac{2}{2 k+1}, g_{k}=\frac{2 k+3}{2 k+7}$, and $h_{k}=-\left(1+g_{k}\right)$.

(2) When $k=N-3, N-2, j=0,1, \ldots, N-4, a_{k j}=0$, and the only nonzero elements of $b_{k j}$ and $c_{k j}$ are

$$
\begin{array}{ll}
b_{N-3,0}=b_{0, N-3}=\frac{3 \sqrt{10}}{35}, & b_{N-3,2}=b_{2, N-3}=-\frac{\sqrt{2}}{105} \\
b_{N-2,1}=b_{1, N-2}=\frac{\sqrt{14}}{63}, & b_{N-2,3}=b_{3, N-2}=-\frac{\sqrt{22}}{693} \\
c_{N-3,0}=c_{0, N-3}=\frac{\sqrt{10}}{5}, & c_{N-2,1}=c_{1, N-2}=\frac{\sqrt{14}}{7} .
\end{array}
$$


(3) When $k=N-3, N-2, j=N-3, N-2$, the only nonzero elements of $a_{k j}, b_{k j}$, and $c_{k j}$ are

$$
\begin{array}{ll}
a_{N-3, N-3}=18, & a_{N-2, N-2}=150, \\
b_{N-3, N-3}=12 / 5, & b_{N-2, N-2}=20 / 21, \\
c_{N-3, N-3}=6, & c_{N-2, N-2}=10 .
\end{array}
$$

From the fact that $\varphi_{i}^{\prime}( \pm 1)=0(i=0,1, \ldots, N-4), \varphi_{N-3}^{\prime}( \pm 1)=\mp 3$, and $\varphi_{N-2}^{\prime}( \pm 1)=-5$, we immediately obtain the following lemma.

Lemma 4.2 Let $p_{i j}=\varphi_{j}^{\prime}(-1) \varphi_{i}^{\prime}(-1), q_{i j}=\varphi_{j}^{\prime}(1) \varphi_{i}^{\prime}(1)$, then

$$
p_{i j}=\left\{\begin{array}{ll}
9, & i=j=N-3, \\
25, & i=j=N-2, \\
p_{j i}=-15, & i=N-2, j=N-3, \\
0, & \text { otherwise, }
\end{array} \quad q_{i j}= \begin{cases}9, & i=j=N-3, \\
25, & i=j=N-2, \\
q_{j i}=15, & i=N-2, j=N-3 . \\
0, & \text { otherwise. }\end{cases}\right.
$$

Next we will build the matrix formulation for the discrete equation (2.5) by means of the tensor product.

- Case $d=2$.

It is easy to see that $X_{N}=\operatorname{span}\left\{\phi_{i}(x) \phi_{j}(y): i, j=0,1, \ldots, N-2\right\}$. Thus, we shall seek

$$
w_{N}=\sum_{i, j=0}^{N-2} w_{i j} \phi_{i}(x) \phi_{j}(y)
$$

Put

$$
\boldsymbol{W}=\left(\begin{array}{cccc}
w_{00} & w_{01} & \cdots & w_{0, N-2} \\
w_{10} & w_{11} & \cdots & w_{1, N-2} \\
\vdots & \vdots & \cdots & \vdots \\
w_{N-2,0} & w_{N-2,1} & \cdots & w_{N-2, N-2}
\end{array}\right)
$$

By denoting the column vectors of $\boldsymbol{W}$ by $\overline{\boldsymbol{w}}$, plugging the (4.1) into (2.5), taking $v_{N}$ through all the basis functions in $X_{N}$, and using tensor product, we can simplify the Legendre-Galerkin approximation to the system (2.5) in two-dimensional case as follows:

$$
\mathbb{A} \overline{\boldsymbol{w}}=\chi_{N} \mathbb{B} \overline{\boldsymbol{w}}
$$

where $\mathbb{A}=B \otimes A+A \otimes B+2 C \otimes C, \mathbb{B}=(P+Q) \otimes B+B \otimes(P+Q), A=\left\{a_{i j}\right\}_{i, j=0}^{N-2}, B=\left\{b_{i j}\right\}_{i, j=0}^{N-2}$, $C=\left\{c_{i j}\right\}_{i, j=0}^{N-2}, P=\left\{p_{i j}\right\}_{i, j=0}^{N-2}, Q=\left\{q_{i j}\right\}_{i, j=0}^{N-2}$, and $\otimes$ denotes the notation of tensor product of matrix.

- Case $d=3$.

It is easy to see that $X_{N}=\operatorname{span}\left\{\phi_{i}(x) \phi_{j}(y) \phi_{k}(z): i, j, k=0,1, \ldots, N-2\right\}$. Hence, we shall look for

$$
w_{N}=\sum_{i, j, k=0}^{N-2} w_{i j k} \phi_{i}(x) \phi_{j}(y) \phi_{k}(z)
$$


Put

$$
\boldsymbol{W}^{k}=\left(\begin{array}{cccc}
w_{00}^{k} & w_{01}^{k} & \cdots & w_{0, N-2}^{k} \\
w_{10}^{k} & w_{11}^{k} & \cdots & w_{1, N-2}^{k} \\
\vdots & \vdots & \cdots & \vdots \\
w_{N-2,0}^{k} & w_{N-2,1}^{k} & \cdots & w_{N-2, N-2}^{k}
\end{array}\right) .
$$

By denoting the column vectors of $\boldsymbol{W}^{k}$ by $\overline{\boldsymbol{w}}^{k}(j=1,2, \ldots, N-2)$, plugging the (4.3) into (2.5), taking $v_{N}$ through all the basis functions in $X_{N}$, and using tensor product, we can simplify the Legendre-Galerkin approximation to the discrete equation (2.5) in the threedimensional case as follows:

$$
\mathbb{A} \overline{\boldsymbol{w}}^{k}=\chi_{N} \mathbb{B} \overline{\boldsymbol{w}}^{k},
$$

where $\mathbb{A}=B \otimes B \otimes A+B \otimes A \otimes B+A \otimes B \otimes B+2 B \otimes C \otimes C+2 C \otimes B \otimes C+2 C \otimes C \otimes B$, $\mathbb{B}=(P+Q) \otimes B \otimes B+B \otimes(P+Q) \otimes B+B \otimes B \otimes(P+Q)$.

Based on (4.2) and (4.4) we can efficiently compute the approximative eigenvalues of (1.1)-(1.3) on the rectangle domain and cubic one, respectively.

\section{Numerical examples}

We now carry out some numerical experiments to compute the eigenvalues of (2.1) on $\bar{\Theta}=[0, \pi / 2]^{2}$ and $\bar{\Theta}=[0, \pi / 2]^{3}$, respectively. By using finite element methods, [13] computed the first four eigenvalues of $(2.1)$ on $\bar{\Theta}=[0, \pi / 2]^{2}$ and gave the bounds of the exact eigenvalues as follows:

$$
\begin{array}{ll}
\chi_{1} \in(2.2126268,2.2126974), & \chi_{2} \in(4.4156245,4.4161723), \\
\chi_{3} \in(4.4156245,4.4161723), & \chi_{4} \in(6.0803290,6.0817236) .
\end{array}
$$

However, as far as we know, there has appeared no report of numerical results on threedimensional regions. In this article, we employ the Legendre polynomials to formulate the basis functions such that the matrices in the discrete variational formulation are sparse, which makes it efficient and easy for us to compute eigenvalues of (2.1) on threedimensional regions. We operate our programs in MATLAB. The numerical results are listed in Table 1 and Table 2, where DOF denotes the degree of freedom (number of unknowns).

We can see that the eigenvalues in Table 1 have at least ten-digit accuracy with $N=25$, i.e., $\mathrm{DOF}=576$. As a comparison, we observe that the eigenvalues obtained by using finite element in [13] only have four-digit accuracy for $h=\sqrt{2} \pi /(80)$, i.e., DOF $=6,244$.

Table 1 The approximative eigenvalues on $\bar{\Theta}=[0, \pi / 2]^{2}$

\begin{tabular}{lllll}
\hline $\boldsymbol{N}$ & DOF & $\boldsymbol{\chi}_{\mathbf{1}}$ & $\boldsymbol{\chi}_{\mathbf{2}}$ & $\boldsymbol{\chi}_{\mathbf{4}}$ \\
\hline 15 & 196 & 2.212697396 & 4.416172185 & 6.081723134 \\
20 & 361 & 2.212697395 & 4.416172142 & 6.081722889 \\
25 & 576 & 2.212697395 & 4.41617214 & 6.081722871 \\
30 & 841 & 2.212697395 & 4.41617214 & 6.081722871 \\
\hline
\end{tabular}


Table 2 The approximative eigenvalues on $\bar{\Theta}=[0, \pi / 2]^{3}$

\begin{tabular}{rrlll}
\hline $\boldsymbol{N}$ & \multicolumn{1}{c}{ DOF } & $\chi_{\mathbf{1}}$ & $\chi_{\mathbf{2}}$ & $\boldsymbol{\chi}_{\mathbf{5}}$ \\
\hline 10 & 729 & 2.989297949 & 4.966408489 & 6.533085863 \\
15 & 2,744 & 2.989297401 & 4.966394295 & 6.532973331 \\
20 & 6,859 & 2.989297388 & 4.966394153 & 6.532972744 \\
25 & 13,824 & 2.989297388 & 4.966394148 & 6.532972712 \\
\hline
\end{tabular}

It also can be seen from Table 2 that the eigenvalues have at least eight-digit accuracy with $N=20$. By computing, for the problem $(2.1)$ on $\bar{\Theta}=[0, \pi / 2]^{3}$, we also see that $\chi_{1}$ is a simple eigenvalue, $\chi_{2}$ and $\chi_{5}$ are all eigenvalues with multiplicity 3 .

\section{Conclusions}

In this study, we have establish an efficient spectral-Galerkin formulation for the fourthorder Steklov equation with boundary eigenvalue. By analyzing the error formulas of projective operators and adopting the compact-operator spectral method, we have derived the error formulas of approximative eigenvalues and eigenfunctions. Then we have formulated a suitable set of basis functions included in $H_{0}^{1}(\Theta) \cap H^{2}(\Theta)$ and built the matrix formulation for the discrete variational scheme by means of the tensor product. In this way, we can efficiently solve the discrete system and obtain highly accurate approximative eigenvalues. We have provided the numerical examples in rectangle domain and cubic one and the satisfactory results obtained show that our method is very effective. In this study, we confined our focus to the cases in the rectangle domain and the cubic one. In fact, the technique used in this article could be expanded to more general domains by adopting a spectral-element technique.

Competing interests

The authors declare to have no competing interests.

Authors' contributions

The authors wrote, read, and approved the final manuscript.

\section{Author details}

${ }^{1}$ School of Mathematical Science, Guizhou Normal University, Huaxi District, Guiyang, 550025, China. ${ }^{2}$ School of Mathematics and Physics, North China Electric Power University, No. 2, Bei Nong Road, Changping District, Beijing, 102206, China.

\section{Acknowledgements}

This research was supported by National Science Foundation of China grant 11271127, 11671106, and 11661022.

Received: 12 July 2016 Accepted: 31 August 2016 Published online: 07 September 2016

\section{References}

1. Bramble, JH, Osborn, JE: Approximation of a Steklov eigenvalue problem of non-self adjoint second order elliptic operators. In: Aziz, AK (ed.) Mathematical Foundations of the Finite Element Method with Application to PDE. Academic Press, San Diego (1972)

2. Andreev, AB, Todorov, TD: Isoparametric finite-element approximation of a Steklov eigenvalue problem. IMA J. Numer. Anal. 24, 309-322 (2007)

3. Armentano, MG, Padra, C: A posteriori error estimates for the Steklov eigenvalue problem. Appl. Numer. Math. $\mathbf{5 8}$ 593-601 (2008)

4. Yang, YD, Li, Q, Li, SR: Nonconforming finite element approximation of the Steklov eigenvalue problem. Appl. Numer. Math. 59, 2388-2401 (2009)

5. Lin, Q, Xie, HH: Nonconforming finite element approximations of the Steklov eigenvalue problem and its lower bound approximations. Appl. Math. 58, 129-151 (2013)

6. Li, Q, Yang, YD: A two-grid discretization scheme for the Steklov eigenvalue problem. J. Appl. Math. Comput. 36, 129-139 (2011)

7. $\mathrm{Bi}, \mathrm{H}, \mathrm{Yang}, \mathrm{YD}$ : A two-grid method of the non-conforming Crouzeix-Raviart element for the Steklov eigenvalue problem. Appl. Math. Comput. 217, 9669-9678 (2011) 
8. Li, MX, Lin, Q, Zhang, SH: Extrapolation and superconvergence of the Steklov eigenvalue problems. Adv. Comput. Math. 33, 25-44 (2010)

9. Tang, WJ, Guan, Z, Han, HD: Boundary element approximation of Steklov eigenvalue problem for Helmholtz equation. J. Comput. Math. 16, 165-178 (1998)

10. Cao, LQ, Zhang, L, Allegretto, W, Lin, YP: Multiscale asymptotic method for Steklov eigenvalue equations in composite media. SIAM J. Numer. Anal. 51(1), 273-296 (2013)

11. Zhang, $X Q$, Yang, YD, Bi, H: Spectral method with the tensor-product nodal basis for the Steklov eigenvalue problems. Math. Probl. Eng. 2013, 650530 (2013)

12. Gazzola, F, Sweers, G: On positivity for the biharmonic operator under Steklov boundary conditions. Arch. Ration. Mech. Anal. 188, 399-427 (2008)

13. Bi, H, Ren, SX, Yang, YD: Conforming finite element approximations for a fourth-order Steklov eigenvalue problem. Math. Probl. Eng. 2011, 873152 (2011). doi:10.1155/2011/873152

14. Shen, J, Tang, T, Wang, L: Spectral Methods Algorithms, Analysis and Applications. Springer, Heidelberg (2011)

15. Luo, ZD: A high accuracy numerical method based on spectral theory of compact operator for biharmonic eigenvalue equations. J. Inequal. Appl. 2016, 77 (2016)

16. Babuska, I, Osborn, J: Eigenvalue problems. In: Ciarlet, PG, Lions, JL (eds.) Handbook of Numerical Analysis, Finite Element Methods (Part 1), vol. 2, pp. 640-787. Elsevier, Amsterdam (1991)

17. Osborn, JE: Spectral approximation for compact operators. Math. Comput. 29(131), 712-725 (1975)

18. Shen, J: Efficient spectral-Galerkin method I. Direct solvers for second- and fourth-order equations using Legendre polynomials. SIAM J. Sci. Comput. 15(6), 1489-1508 (1994)

\section{Submit your manuscript to a SpringerOpen ${ }^{\circ}$ journal and benefit from:}

- Convenient online submission

Rigorous peer review

- Immediate publication on acceptance

- Open access: articles freely available online

- High visibility within the field

- Retaining the copyright to your article 